\title{
Research on Application of Anterolateral Thigh Perforator Flap to Repair Wounds of Hand and Foot
}

\author{
Yongming Tao, Yanhong Liu ${ }^{\text {a, * }}$ \\ Lanzhou Orthopedics Hospital of Traditional Chinese Medicine Lanzhou 730010 China. \\ *, a Liuyanhong@163.com
}

\begin{abstract}
Keywords: Anterior-medial side of thigh; perforated flap; Transplantation; free-graft; Repairing;
\end{abstract} Hand and foot and wound.

\begin{abstract}
Purpose: Analysis the Clinical curative effect of the therapy that transfer the Perforator Flaps free-graft of anterior-medial side of thigh for foot and hand trauma. Methods: We selected 24 hands or foot trauma cases, which were treated with therapy that transfer the Perforator Flaps free-graft of anterior-medial side of thigh, from July 2014 to December 2017, studied the curative effect of the surgical application, and discussed the situation of postoperative vasospasm and the recovery of the skin flap. Result: In the study, there were no vascular crisis after our operation and all flaps survived. After the operation, we take a 3-6months follow up of our patients, some of their skin flap was slightly swollen, but the texture was good. 10 of them were with good anastomosis in their operation area and began to have protective sensation in the skin flap. All the functions were not affected. Conclusion: The clinical curative effect of the therapy that transfer the Perforator Flaps free-graft of anterior-medial side of thigh for foot and hand trauma is remarkable, we can popularize it widely.
\end{abstract}

\section{Introduction}

In daily life, because the hands and feet need to be exposed to more objects, the incidence of skin defect is higher [1-2]. Chronic ulcer, soft tissue skin necrosis, burns, car accident, mechanical injury and so on, are all important causes of hand and foot trauma [3-4]. In general, patients with hand and foot trauma will be accompanied by the appearance of external tendons and osteopenia. If we only use conventional suture technique to treat and repair it, it is difficult to obtain the ideal therapeutic effect [5-6]. In recent years, with the continuous deepening of clinical research and the development of microsurgical technique, the perforated flap has been widely used in the repair of hand and foot trauma [7-8]. Medial gastrocnemius perforator flaps, anterolateral thigh perforator flaps, inferior epigastria artery perforator flaps, anterior-medial side of thigh perforator flaps are several common types of free-graft perforated flap. We analysis the Clinical curative effect of the therapy that transfer the Perforator Flaps free-graft of anterior-medial side of thigh for foot and hand trauma in this article, reported as follow.

\section{Materials and Methods}

\subsection{Clinical Data.}

We randomly selected 24 hands or foot trauma cases, which were treated with therapy that transfer the Perforator Flaps free-graft of anterior-medial side of thigh, from July 2014 to December 2017. The age of the patients were 33 to 48 years old, with an average age of $(45.69+2.62)$. The defect area was between $(4.1 \times 6.0) \mathrm{cm}-(9.1 \times 13.0) \mathrm{cm} ; 20$ males and 4 females; 14 cases of them were foot defects, 10 of them were hand - back or palmar defects. There were 2 cases, 2 cases, 8 cases and 12 cases in patients with chronic ulcer, burn with partial necrosis, crush injury and machine injury. This study was approved by the hospital ethics committee, and all patients have signed the informed consent form voluntarily. All the patients, after the second phase of debridement, had been treated with the therapy that transfer the Perforator Flaps free-graft of anterior-medial side of thigh for foot and hand trauma. 


\subsection{Method.}

After the successful anesthetic, we operate a thorough debridement treatment to the trauma area, measure the size of the wound and perform a template sampling. We performed routine anatomy of the associated nerve and blood vessels in the trauma area. If the patient is with hand trauma, we should dissect the cutaneous nerve, radial arteriovenous vein or ulnar arteriovenous vein, using the trauma area as the basis. If it is a foot trauma, we need to dissect the cutaneous nerve and the dorsal artery. The design method of skin flap is: First, we perform a color Doppler ultrasonography to the patients to understand the blood flow situation. The fulcrum of the perforator Flaps of anterior-medial side of thigh should be adjacent to the middle point between the anterior superior iliac spines to the medial condyle of the femoral condyle. Then, we will use the fulcrum as the benchmark to make a reasonable design of the perforated skin flap based on the size and shape of the plane, make sure the flap is in the middle of the thigh. Then, we cut the skin along the lateral edge of the flaps, in which we can extend the incision lengthwise according to the actual situation. From the deep surface of the fascia Lata, we lift the flaps to the medial side to identify the sartorius muscle, the vastus medialis, and the femoral rectus muscle effectively to find the superficial perforating vessels near the muscle triangle. In case of need, we can adjust the design of the flap according to the direction and position of the vessel. Open the flap along the edge, lift the flap, and gently lift the sartorius on the inside. According to the specific requirements of the vascular pedicle length in the trauma area, we dissected the proximal end of the perforated blood vessel along the shallow-point. In this position, the perforated vein and the artery are associated with each other, and we can continue to separate and process along the interval of the muscle, until we finally get to the starting point of the vascular pedicle. In this study, when we were resecting the flap and looking through the perforator, we found that there were 8 patients whose perforating branches of the perforator flaps are derived from femoral artery and 16 of whose are derived from the medial branch from the lateral branch of the lateral femoral artery. When we cut the edge of the flap, we can find the anterior cutaneous nerve branch in the flaps area. When we are dissociating the flap, we should take the epithelial nerve to match the nerves in the trauma area. Cut off the vasculature of the flap, Transplantation of flap to the trauma area, suture the edge of it, and fix it properly. Taking surgical microscopy as an assistant, we anastomose the cutaneous nerve and the arterial vein with those in trauma area. If the blood vessels are found to be in a free flow, and the flap is well delivered, we can stitch the wounds, then we could graft or sutured the donor site.

\section{Result}

In the study, there were no vascular crisis after our operation and all flaps survived. After the operation, we take a 3-6months follow up of our patients, some of their skin flap was slightly swollen, but the texture was good. 10 of them were with good anastomosis in their operation area and began to have protective sensation in the skin flap. All the functions were not affected.

\section{Typical Case Analysis}

Mr. Li, age 48, male, the car accident caused his right foot swelling and bleeding. He got into the hospital within 2 hours and we diagnosed as severe compression injury to the right foot. After emergency debridement and repair, his foot was left with a wound $(6.0 \times 9.0) \mathrm{cm}$. The tendons are exposed, and there are defects in the soft tissue of the skin. The treatment we performed is free transplantation of the left iliac inguinal flap, which was used to repair the wound surface effectively. Taking Routine debridement treatment for the affected area, we dissected the lateral cutaneous nerve and the dorsal leg. After reasonable design of the skin flap according to the actual condition of the patient, we examined the donor area carefully. The results showed that the left lateral iliac artery was slender and the outer diameter was only $0.2 \mathrm{~mm}$. It is too risky to treat with free transplantation prosthesis. So, we finally decided to use the left femoral anterior medial perforation flap. First, design the skin flap in front of the left thigh, and slice the skin along the lateral edge of the flap. Then, we cut 
the skin along the lateral edge of the flaps. From the deep surface of the fascia Lata, we lift the flaps to the medial side to identify the sartorius muscle, the vastus medialis, and the femoral rectus muscle effectively to find the superficial perforating vessels near the muscle triangle. In case of need, we can adjust the design of the flap according to the direction and position of the vessel. Open the flap along the edge, lift the flap, and gently lift the sartorius on the inside. According to the specific requirements of the vascular pedicle length in the trauma area, we dissected the proximal end of the perforated blood vessel along the shallow-point. In this position, the perforated vein and the artery are associated with each other, and we can continue to separate and process along the interval of the muscle, until we finally get to the starting point of the vascular pedicle. In this study, when we were resecting the flap and looking through the perforator, we found that there were 8 patients whose perforating branches of the perforator flaps are derived from femoral artery and 16 of whose are derived from the medial branch from the lateral branch of the lateral femoral artery. When we cut the edge of the flap, we can find the anterior cutaneous nerve branch in the flaps area. When we are dissociating the flap, we should take the epithelial nerve to match the nerves in the trauma area. Cut off the vasculature of the flap, Transplantation of flap to the trauma area, suture the edge of it, and fix it properly. Taking surgical microscopy as an assistant, we anastomose the cutaneous nerve and the arterial vein with those in trauma area. If the blood vessels are found to be in a free flow, and the flap is well delivered, we can stitch the wounds, then we could graft or sutured the donor site. After the operation, the patient needs to receive anti-infective, antispasmodic, anticoagulant treatment. After 2 months of the operation, the skin flap was observed to be good. 6 months after the main operation, we performed an operation to improve the shape of the flap and obtained a satisfactory appearance of the flap.

\section{Conclusion}

Hand and foot trauma is very common in clinical medicine. At present, we usually use various flaps with pedicles for retrograde transfer repair. However, when the wound is larger, the method has some limitations [9-10]. In recent years, with the continuous deepening of clinical research and the development of microsurgical technique, the perforated flap has been widely used in clinical. The Blood supply artery of anterior-medial side of thigh flap is located at center of the vastus medialis triangle that construct by the vastus medialis muscle, the rectus femoris, and the sartorius muscle, it has a relatively constant position [11-12]. The femoral artery, the superficial femoral artery and the lateral femoral artery are the three main sources of the blood supply of the flap. The external diameter of the femoral artery is between $1.8 \mathrm{~mm}$ and $2.4 \mathrm{~mm}$, company with a vein. The external diameter of femoral artery was $1.5 \mathrm{~mm}$, and the external diameter of the lateral femoral artery was $2.5 \mathrm{~mm}$. In this study, 8 patients' perforating branches were derived from the Femoral artery, 16 of them were derived from the medial branch of the lateral branch of the lateral femoral artery. We have not found those derived from superficial femoral artery till now.

The perforator flap is a major development of microsurgical flap transplantation. This is highly consistent with the principle of tissue transplantation, it is not necessary to anatomize the source of the perforated flap of free transplantation, therefore the operation process is simple, arbitrary and flexible. It is an ideal repair method of soft tissue defect after trauma [13-15]. Compared with the perforated flap with higher frequency in clinical practice, the anterior medial femoral cutaneous flap was less hairy, and the skin was whiter and had a thinner flap. But at the same time, there is a constant vascular anatomy, the blood vessel diameter is thicker, and the blood vessel is longer. Usually, the area can be sutured directly, so the patient does not need to change his position during the operation. At present, the perforated flap and the front lateral flap are widely used in clinic. If there is an operation error or a variation, we considered to dissect the flaps in anterior-medial side of thigh. In the study, there were no vascular crisis after our operation and all flaps survived. After the operation, we take a 3-6months follow up of our patients, some of their skin flap was slightly swollen, but the texture was good. In conclusion, the clinical curative effect of the therapy that transfer the Perforator Flaps free-graft of anterior-medial side of thigh for foot and hand trauma is remarkable, we can 
popularize it widely. But during surgery, we should perform a Doppler ultrasound blood flow test to located perforator vessel of the anterior-medial side of thigh perforator flap near the muscular triangle clearly. During the operation, we first dissect the muscle triangle, find the blood vessels, and adjust the position of the flap if necessary.

The clinical curative effect of the therapy that transfer the Perforator Flaps free-graft of anterior-medial side of thigh for foot and hand trauma is remarkable, we can popularize it widely.

\section{References}

[1]. Chen Shi, Qi Shilling, Yan Yi, et al. Repair the soft tissue defect of the malleolus with a flap in front of free ultrathin femoral skin [J]. Chinese orthopedic surgery journal, 2016, 24(14):1340-1343.

[2]. Song Darian, Li Zen, Zhou Xiao, etc. Free shares before the medial perforators flap to repair defects of oral cavity cancer radical surgery and relay femoral anterolateral perforators flap donor area wound effect [J]. Chinese journal of burns, 2017, 33 (2): 72-76.

[3]. Mo Yong Jun, Tan Haitao, Yang Keqin, et al. The application of CTA 3d reconstruction technique in front lateral femoral cutaneous flap transplantation [J]. Chinese orthopedic surgery journal, 2016, 24(6):570-573.

[4]. Tang Yuan, Tang Jug $\mathrm{Yu}, \mathrm{Wu}$ Panfeng, et al. The treatment of the lateral femoral artery of the lateral femoral artery for the treatment of osteomyelitis after tibial trauma [J]. Chinese journal of modern medicine, 2017, 27(8):76-79.

[5]. Shi Quant, Wei Sarong, Jin Wenhu, et al. The feasibility and effect of perforating flaps in the front lateral perforation of the femoral cutaneous flap of the superficial iliac artery perforation [J]. China burn journal, 2017, 33(5):309-311.

[6]. From Zhuhai, Pan Yuliana Zhao, Xue Shan, etc. Bridge iliac and femoral anterolateral perforators flap of cutaneous nerve composite tissue flap transplantation to repair the foot bone soft tissue defect in the case of [J]. Chinese journal of plastic surgery, 2016, 32 (5): 389-390.

[7]. Xian Wending, Wang Haiden, Jiang Xinming, et al. Anterior lateral perforation of the hemodynamic bridge with a flap to repair the soft tissue defect of the palm skin with blood supply disorder [J]. Chinese journal of clinical anatomy, 2016, 34(1):24-27.

[8]. You Joshing, Liu Haiti, Li Hongye, et al. Repair of the hand wound in the front lateral perforation of the anterior lateral perforation [J]. Chinese journal of hand surgery, 2016, 32(1):75-76.

[9]. Liu Mingling, Liu Liang, Xiao Xiangjun, et al. Analysis of the curative effect of the anterior lateral perforation of free femoral skin flap in repairing the deep ulcer of the limbs [J]. Journal of central south medical science, 2017, 45(1):98-100.

[10]. Zhao Juneau, Fu Don Sheng, Chen Jajuan, et al. Repair effect of the lateral perforation of the lateral femoral flap on wrist, foot and soft tissue defects [J]. Modern instruments and medical treatment, 2017, 23(3):93-94.

[11]. Zoo Yon tong, Liu Xiao Jun, Zhu ping, et al. Application of the anterior lateral perforation of the femoral flap in skin defect repair after severe trauma of extremities [J]. Hainan medicine, 2017, 28(11):1854-1856.

[12]. Waning, Wei Sarong, Jin Wenhu, et al. Microchipping of the lateral arterial descending branch of the lateral femoral artery to repair the complex wound surface of limbs [J]. Chinese plastic surgery journal, 2016, 32(4):303-305. 
[13]. Zhao Gangue, Zhang Dewey, Hu Yonggang, et al. The application of the lateral dissection of the anterior lateral flap of thin femoral head in the reconstruction of the soft tissue of the ankles [J]. Chinese journal of aesthetic plastic surgery, 2017, 28(2):86-89.

[14]. Zhang Wan Feng Zhang Xiao Feng, GAO Qi fang, etc. The scalp type axial vascular network skin flap or the femoral anterolateral perforators of fascia flap repair of skin defect after radical resection of malignant tumors in patients [J]. Chinese journal of burns, 2017, 33 (8): 491-496.

[15]. Zhou Wei, Zhou Netcong, Tang Juyu, et al. Postoperative management of the repair of soft tissue defect in limbs with lateral femoral artery of the lateral femoral artery [J]. Chinese journal of microsurgery, 2017, 40(1). 